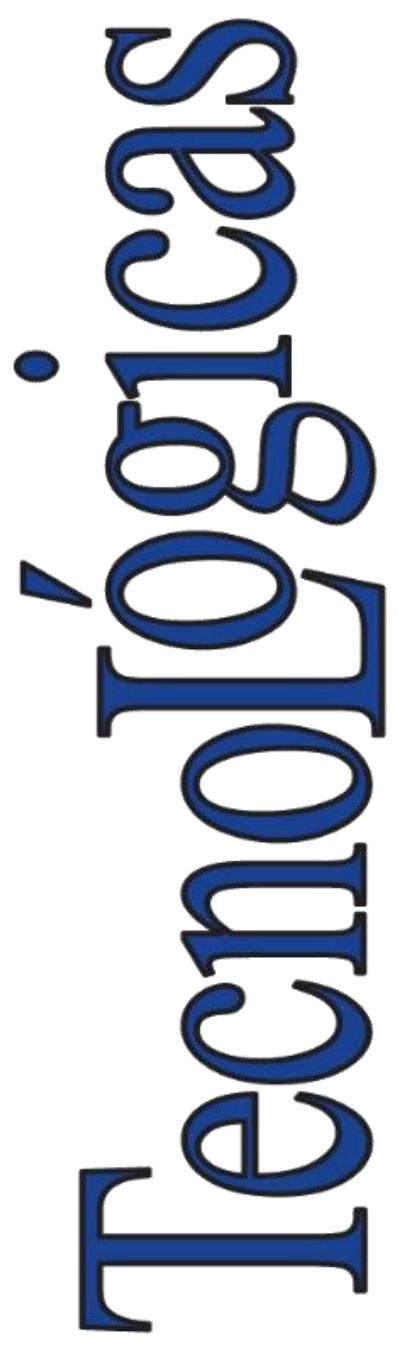

ISSN-p: 0123-7799 ISSN-e: $2256-5337$

Vol. 24, nro. 52, e2144, 2021

Recibido: 10 junio 2021 Aceptado: 11 noviembre 2021 Disponible: 17 diciembre 2021

CInstituto Tecnológico Metropolitano Este trabajo está licenciado bajo una Licencia Internacional Creative Commons Atribución (CC BY-NC-SA)

\section{Análisis de la precipitación y la evaporación en el Orinoco colombiano según los modelos climáticos regionales del experimento CORDEX-CORE}

\author{
Analysis of Precipitation and Evaporation in the \\ Colombian Orinoco According to the Regional Climate \\ Models of the CORDEX-CORE Experiment
}

\author{
(D) Carolina Florian-Vergara ${ }^{1}$; \\ (iD) Hernán D. Salas ${ }^{2}$; \\ iD Alejandro Builes-Jaramillo ${ }^{3}$ \\ ${ }^{1}$ Institución Universitaria Colegio Mayor de Antioquia \\ Medellín-Colombia, \\ carolina.florian@colmayor.edu.co \\ 2 Institución Universitaria Colegio Mayor de Antioquia \\ Medellín-Colombia, \\ hernan.salas@colmayor.edu.co \\ ${ }^{3}$ Institución Universitaria Colegio Mayor de Antioquia \\ Medellín-Colombia, \\ luis.builes@colmayor.edu.co
}

Cómo citar / How to cite

C. Florian-Vergara; H. D. Salas; A. Builes-Jaramillo, "Análisis de la precipitación y la evaporación en el Orinoco colombiano según los modelos climáticos regionales del experimento CORDEX-CORE”, TecnoLógicas, vol. 24, nro. 52, e2144, 2021.

https://doi.org/10.22430/22565337.2144 


\title{
Resumen
}

Con el fin de representar la precipitación y evaporación total mensual en una cuenca hidrográfica del Orinoco colombiano, este trabajo evaluó la capacidad de los modelos climáticos regionales incluidos en el Experimento regional coordinado de reducción de escala (CORDEX-CORE). Para ello, complementariamente, se incluyeron datos de precipitación y evaporación total de fuentes como Climate Hazards Center InfraRed Precipitation with Station data (CHIRPS), el reanálisis atmosférico (ERA5), Global Precipitation Climatology Center (GPCC) y Global Land Evaporation Amsterdam Model (GLEAM). Las comparaciones entre los ensambles de los modelos y las observaciones se hicieron utilizando métodos gráficos y métodos cuantitativos, entre ellos: diagramas de cajas, porcentajes de sesgo, eficiencia de Nash-Sutcliffe, entre otros. Los resultados evidencian que los valores promedio de precipitación están adecuadamente representados, en términos de su temporalidad y magnitud, por el ensamble del modelo RegCM, mientras que los valores promedio de evaporación total están mejor representados por el ensamble del modelo REMO en términos de la temporalidad, más no en su magnitud. Por otra parte, las estimaciones de caudal de largo plazo evidencian que los valores de evaporación total proporcionados por los modelos permiten una adecuada estimación del caudal promedio de largo plazo, pero no la adecuada estimación del ciclo anual de caudales. Este trabajo es pionero en la evaluación de los datos de precipitación y evaporación total mensual suministrados por CORDEX-CORE en el Orinoco colombiano, sienta precedentes para la incorporación de datos de modelos regionales para fines hidrológicos en zonas poco instrumentadas del país, y es el primer paso hacia la evaluación de escenarios regionalizados de cambio climático.

\section{Palabras clave}

Precipitación mensual, evaporación mensual, balance hídrico, comparación de modelos, modelos climáticos regionales, evaluación de desempeño.

\begin{abstract}
We evaluate the capacity of the Regional Climate Models, included in the regional scale reduction experiment (CORDEX-CORE), to represent the monthly precipitation and evaporation in a watershed of the Colombian Orinoco region. In addition, we use precipitation and evaporation data from sources such as Climate Hazards Center InfraRed Precipitation with Station data, ERA5 atmospheric reanalysis, Global Precipitation Climatology Center and Global Land Evaporation Amsterdam Model. The comparisons between the assemblies of the RCMs and the observations were done using graphical and quantitative methods, among them: box plots, percentages of bias, Nash-Sutcliffe efficiency, among others. Our results allow concluding that the average precipitation values are adequately represented, in terms of their temporality and magnitude, by the assembly of the RegCM model while the average values of total evaporation are better represented by the assembly of the REMO model in terms of temporality, but not in its magnitude. Furthermore, the long-term flow estimation shows that the total evaporation values provided by the RCMs allow an adequate estimation of the long-term average flow, but not the adequate estimation of the annual flow cycle. This work is a pioneer in the evaluation of the monthly precipitation and evaporation data provided by CORDEX-CORE in the Colombian Orinoco, sets precedents for the incorporation of regional models data for hydrological purposes in poorly instrumented areas of the country, and is the first step towards the evaluation of regional climate change scenarios.
\end{abstract}

\section{Keywords}

Monthly precipitation, Monthly evaporation, Water Balance, Model Comparison, Regional Climate Models, Performance assessment. 


\section{INTRODUCCIÓN}

El territorio continental de Colombia está conformado por las regiones naturales: Caribe, Pacífico, Andina, Amazonía y Orinoquía. Ésta última recibe su nombre debido a que la mayor parte de sus ríos se encuentran en la cuenca del río Orinoco y está compuesta por los departamentos de Arauca, Casanare, Meta y Vichada, abarcando el $18 \%$ del territorio continental nacional, limitando al norte y al este con Venezuela, al sur con la región Amazónica y al oeste con la región Andina [1]. La Orinoquia cuenta con un régimen de lluvias unimodal, un periodo húmedo que inicia en abril y finaliza en noviembre, seguido de un periodo de sequía que va desde diciembre hasta marzo [2]. Además, la región exhibe una precipitación anual que no es uniforme en toda su área, ya que lugares como el Piedemonte y Villavicencio exhiben más de dos veces la precipitación media.

Mientras el Orinoco experimenta su temporada seca (noviembre-marzo), la región de bajas presiones atmosféricas, conocida como zona de convergencia intertropical (ZCIT), migra hacia el sur, intensificando los vientos alisios sobre el noreste de Venezuela [3]. Esta migración se relaciona con un gradiente de presión establecido entre el Alto Subtropical del Atlántico Norte (NASH, por sus siglas en inglés) y el Amazonas, permitiendo así que los vientos alisios entren desde el noreste hasta el suroeste de Venezuela, creando un patrón de circulación conocido como el chorro de bajo nivel del Orinoco (OLLJ, por sus siglas en inglés), el cual influye directamente en el régimen de precipitaciones en la cuenca del río Orinoco [4][6].

Los modelos climáticos globales (MCG) permiten representar e investigar la dinámica climática global y pretenden simular la respuesta a cambios de patrones en variables del sistema climático, tales como el incremento de los gases de efecto invernadero o la radiación [7]. Estos modelos representan los procesos biogeoquímicos de la atmósfera, el océano y la superficie terrestre, así como sus interacciones y funciones de retroalimentación [8], [9].

Los MCG representan el clima utilizando una rejilla tridimensional sobre el globo terráqueo, normalmente con una resolución horizontal superior a los $100 \mathrm{~km}$, mientras la atmósfera se representa a través de 10 a 20 capas verticales y 30 para el océano. Esta resolución es muy gruesa para observar detalladamente los procesos de la superficie terrestre y del clima de manera directa en una región o lugar específico [10]-[12]. Por lo tanto, las limitaciones dadas por la baja resolución espacial de los MCG hacen necesario reducir la escala para obtener información aproximada a las condiciones reales de una región, razón por la que, metodologías como la regionalización estadística y/o dinámica, son implementadas mediante modelos de mayor resolución espacial o modelos climáticos regionales (MCR), con el fin de representar procesos de manera más detallada que los MCG [7], [8], [13], [14]. Los MCR son la principal técnica de regionalización dinámica, ya que resuelven mejor las propiedades locales de la superficie terrestres, la topografía, las costas, la vegetación y la variabilidad climática regional interna [15].

Dentro de los esfuerzos de modelación regional, se encuentra el Experimento regional coordinado de reducción de escala (CORDEX), cuya misión principal es regionalizar escenarios de cambio climático en el ámbito global y, a su vez, generar un espacio para que la comunidad que esté interesada en esos escenarios pueda compartir información, tecnología, resultados y soporte técnico, generando así una mejor interacción entre los modeladores y las personas interesadas en realizar estudios sobre vulnerabilidad y adaptación al cambio climático [16]. Con el objetivo de coordinar el esfuerzo de la comunidad global de modelación regional surge CORDEX-CORE, en el cual se utilizan dos MCR: i) el Regional Model (REMO), desarrollado por el Instituto Max Planck de Meteorología de Alemania [17] y, ii) el Regional Climate Model (RegCM), desarrollado en el Centro Nacional 
de Investigaciones Atmosféricas de los Estados Unidos y administrado por el Centro Internacional de Física Teórica en Italia [18]. Estos MCR han sido utilizados para evaluar los cambios de sistemas monzónicos considerando diferentes concentraciones de gases de efecto invernadero sobre siete dominios de CORDEX [19], para investigar los cambios proyectados en el balance hídrico atmosférico en dos dominios de CORDEX-CORE [20], para identificar regiones de Suramérica donde los MCR generan un valor agregado en comparación con los MCG [21], y para estudiar la variabilidad espacial y temporal de la temperatura y la precipitación para el periodo histórico y escenarios de cambio climático [22][24].

Los MCR REMO y RegCM son modelos atmosféricos hidrostáticos, que emplean más de veinte niveles para representar la atmosfera y se diferencian, principalmente, en que REMO mantiene las mismas parametrizaciones para todo el planeta, mientras que RegCM emplea diferentes parametrizaciones por dominio [25], [26]. Ambos se encuentran a una desagregación espacial de $25 \mathrm{~km}$ para todas las regiones en las que CORDEX ha distribuido el planeta y cuentan con información de borde a partir de tres MCG los cuales son: el Norwegian Earth System Model (NorESM), desarrollado con el apoyo del Consejo de Investigación Noruego [27]; el MPI-ESM-LR, creado por el Instituto Max Planck de Meteorología de Alemania [28]; y el Hadley Center Global Environment Model versión 2 (HadGEM2), desarrollado por el Centro Hadley en el Reino Unido [29]. Se espera que los resultados de las simulaciones de los MCR en escalas espaciales, cada vez más finas, se conviertan en una herramienta para evaluar la respuesta de las cuencas hidrográficas ante las señales de la variabilidad climática y el cambio climático [30].

La evaluación del desempeño de los MCR se desarrolla por medio de métricas de valor agregado con respecto a los MCG [31], o la comparación directa con fuentes de referencia [32]. Dichas comparaciones permiten que los usuarios finales tomen decisiones informadas sobre la aplicabilidad de los resultados de las reducciones de escala de forma directa o con procesos de corrección de sesgos [33]. En Suramérica se han desarrollado evaluaciones del desempeño en la representación de las variables hidrometeorológicas por parte de los MCR.

Dichos trabajos han mostrado avances en la modelación regional con respecto a la modelación global en la representación de la precipitación o evaporación, dependiendo de la región, temporada o cuenca [22], [24].

La reducción de escala de los MCG mediante los MCR permite diagnosticar, evaluar y gestionar los impactos del cambio climático para la adecuada planificación de los recursos hídricos, la conservación de los recursos naturales, el riego de plantaciones de gran escala, entre otras actividades de los diferentes sectores públicos y privados [33], [34].

Especialmente, en cuencas hidrográficas con poca instrumentación, procesos de gran interés como la precipitación, la evaporación, la infiltración y el flujo subterráneo son difíciles de observar de forma continua, y sin ambigüedades, debido a la heterogeneidad de las cuencas y los procesos hidroclimáticos. Además, dichos procesos requieren de un adecuado monitoreo, evaluación y diagnóstico para modelar adecuadamente sus posibles cambios e impactos en escalas espaciales y temporales más finas [35]-[37].

En este sentido, los MCR ofrecen información sobre variables hidrológicas (precipitación, caudales, entre otras) que pueden ser útiles para evaluar el impacto hidrológico en cuencas hidrográficas considerando escenarios de cambio climático [38]-[40].

Para el caso particular de Colombia, pocos estudios han utilizado información de MCG y MCR para la verificación de balances hídricos de largo plazo y modelación hidrológica considerando escenarios de cambio climático [41]-[43].

Este estudio tiene como objetivo evaluar si los MCR, incluidos en el corte de Suramérica para el experimento CORDEX-CORE, representan adecuadamente la precipitación y la 
evaporación total en el Orinoco colombiano. Adicionalmente busca hacer un análisis comparativo entre observaciones de caudal in-situ y las estimaciones de caudal de largo plazo utilizando información de los MCR. Se evaluará el desempeño de las simulaciones del experimento por primera vez en esta zona, con respecto a las observaciones obtenidas por el Instituto de Hidrología, Meteorología y Estudios Ambientales (IDEAM) y otras bases de datos interpoladas espacialmente para el período de referencia utilizado en el quinto informe de evaluación (AR5) del Grupo Intergubernamental de Expertos sobre el Cambio Climático (IPCC), 1986-2005. Por lo tanto, este trabajo será útil para avanzar en aspectos como: (1) La verificación del desempeño, las simulaciones históricas ofrecidas por los MCR para la precipitación y la evaporación total sobre Colombia; (2) La incorporación de la información suministrada por los MCR para análisis hidrológicos e históricos en zonas con poca instrumentación, mediante el estudio del desempeño de los MCR y la estimación del caudal de largo plazo en la cuenca de análisis; (3) Brindar una herramienta metodológica para la selección de información que sirva para el diagnóstico y modelación de los impactos hidrológicos en cuencas hidrográficas de Colombia considerando la información del experimento CORDEX-CORE.

\section{MÉTODOS Y DATOS}

\subsection{Datos}

Como zona de estudio se seleccionó la cuenca hidrográfica del río Meta, la cual tiene un área de $78072 \mathrm{~km}^{2}$ (Figura 1). Esta zona está definida aguas arriba de la estación limnigráfica Aguaverde (código 35267080) del IDEAM, ubicada en el municipio La Primavera, en el departamento de Vichada. Se tomó información mensual de precipitación y evaporación total a una resolución espacial de $0.25^{\circ}$ x $0.25^{\circ}$ de los MCR REMO2015 [44] y RegCM4 [18]. Para cada MCR (REMO y RegCM) se tomó información de la simulación histórica, en el dominio Suramérica del experimento CORDEX-CORE, con base en los datos de MCG: NorESM1-M [27], MPI-ESM-LR [45] y HadGEM2 [29]. Se verificó la homogeneidad y consistencia de la información hidrológica mediante análisis gráfico y pruebas estadísticas para cambios en la media, varianza y valores atípicos (outliers) [46].

Se tomaron 85 estaciones de precipitación, con resolución temporal mensual y un porcentaje de datos faltantes inferior al $15 \%$ en el periodo de referencia 1986-2005. Se utilizaron los datos de caudal de la estación Aguaverde (código 35267080), para el mismo periodo de referencia. Además, considerando que las series de tiempo seleccionadas tienen un porcentaje de datos faltantes inferior a $15 \%$, dichos datos faltantes fueron llenados utilizando los valores mensuales multianuales [46], [48].

Además, se usó información de precipitación a escala temporal mensual y resolución espacial $0.25^{\circ} \times 0.25^{\circ}$ de las siguientes bases de datos: Climate Hazards Center Infra Red Precipitation with Station (CHIRPS) [49], que ha demostrado representar de forma adecuada la lluvia en Colombia [2] y que interpola información de estaciones de medición en tierra; el reanálisis atmosférico del European Centre for Medium Range Forecast (ECMWF) ERA5, el cual posee menos sesgos en la representación de la precipitación sobre Suramérica [50]; y a $0.5^{\circ} \times 0.5^{\circ}$, información interpolada de estaciones en tierra con control de calidad del Global Precipitation Climatology Center (GPCC) [51]. Como referencia de evaporación total a una resolución espacial de $0.25^{\circ}$ x $0.25^{\circ}$, la información del reanálisis ERA5 y del modelo Global Evaporation Amsterdam Model GLEAM v3 [52]. Como referencia de caudal mensual 
promedio de la cuenca se tomaron las observaciones de la estación Aguaverde del IDEAM y los datos de escorrentía superficial de la reanálisis ERA5.

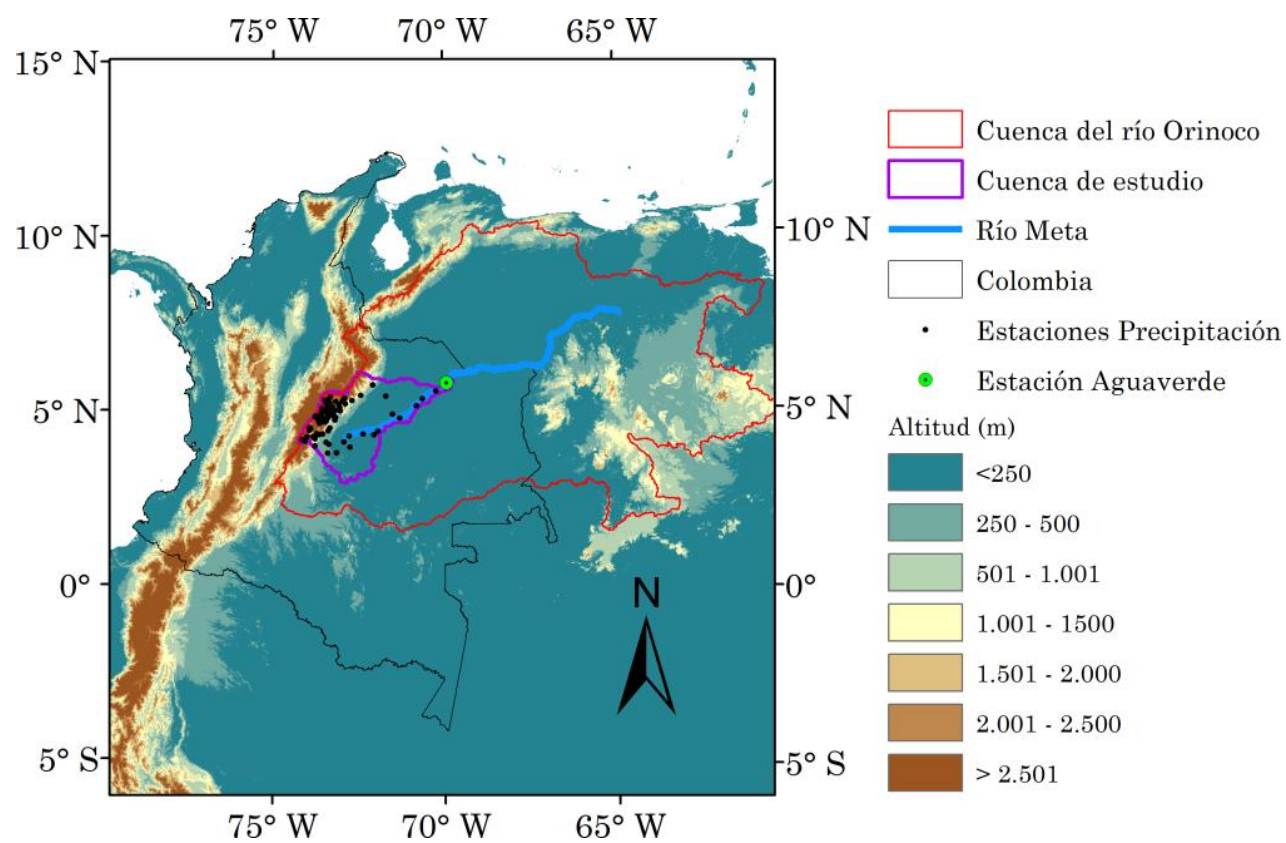

Figura 1. Ubicación de la cuenca del río Orinoco, cuenca de estudio sobre el río Meta y localización de las estaciones de precipitación y estación de caudal empleadas en el presente estudio. Fuente: elaboración propia.

\subsection{Métodos}

Para el análisis comparativo entre las fuentes de información se promediaron los datos de precipitación del IDEAM para todas las estaciones en la cuenca de estudio. Por su parte, con la información obtenida de ERA5, GPCC, CHIRPS y GLEAM se hicieron promedios sobre la cuenca de estudio. Además, para cada uno de los MCR se realizó un ensamble o promedio de las tres simulaciones disponibles, ya que el ensamble permite disminuir las incertidumbres de las regionalizaciones de los MCR [53] y será empleado para evaluar la capacidad de cada MCR para representar el ciclo anual de la precipitación y la evaporación total (valores promedio mensuales multianuales). Las series resultantes de los promedios sobre la cuenca de interés antes mencionada se compararon a través de los métodos que se describen a continuación:

\subsubsection{Métodos gráficos}

El diagrama de caja es una representación gráfica de la distribución y variación de los datos, así como los datos atípicos. La altura de la caja representa el rango intercuartílico entre el primer cuartil (porción inferior) y el tercer cuartil (porción superior). La línea horizontal de la caja representa la mediana mientras que las líneas verticales (bigotes) que salen de la caja se extienden hasta el mínimo y máximo del conjunto de datos. Los valores representados por puntos debajo o por encima de los bigotes se consideran valores atípicos [54]. Complementariamente, como parte de los métodos gráficos, se muestra la comparación entre los ciclos anuales de las fuentes de referencia y de los datos de los MCR. 


\subsubsection{Métodos cuantitativos}

Para una comparación inicial entre los datos de referencia y los resultados de los MCR se utilizaron el coeficiente de correlación de Pearson (R), el coeficiente de determinación (R2), el error típico y la diferencia porcentual. Estas métricas permiten hacer un diagnóstico preliminar sobre la asociación lineal y las diferencias entre los valores de referencia y simulados en términos de su magnitud. Posteriormente, se implementaron para el análisis comparativo las métricas de desempeño compiladas por [55]: porcentaje de sesgo (PBIAS, por su sigla en inglés), relación de desviación estándar de las observaciones (RSR, por su sigla en inglés) y eficiencia de Nash-Sutcliffe (NSE, por su sigla en inglés). Las métricas fueron seleccionadas con base a su robustez, aplicabilidad y uso frecuente para el análisis de modelos y simulaciones hidrológicas. En (1) se define el PBIAS, el cual permite conocer el sesgo porcentual de los datos simulados con relación a las observaciones. Cuando el PBIAS es igual a cero indica que hay una simulación precisa por parte del modelo. Cuando el valor es positivo (negativo), los datos simulados subestiman (sobreestiman) la observación. Esta métrica es utilizada para cuantificar los errores de balances hídricos y provee información sobre el rendimiento de un modelo.

$$
P B I A S=\left[\frac{\sum_{i=1}^{n}\left(Y_{i}^{o b s}-Y_{i}^{s i m}\right) *(100)}{\sum_{i=1}^{n}\left(Y_{i}^{o b s}\right)}\right]
$$

En (2) RSR se define como la relación entre la raíz del error cuadrático medio (RMSE) y la desviación estándar de los datos medidos (STDEVobs). El valor óptimo es cero e indica que la simulación del modelo es perfecta, sin variación entre los datos observados y simulados. Entre menor sea el RSR, mejor es el rendimiento del modelo.

$$
R S R=\frac{R M S E}{S T D E V_{o b s}}=\frac{\left[\sqrt{\sum_{i=1}^{n}\left(Y_{i}^{o b s}-Y_{i}^{\text {sim }}\right)^{2}}\right]}{\left[\sqrt{\sum_{i=1}^{n}\left(Y_{i}^{\text {obs }}-Y_{i}^{\text {mean }}\right)^{2}}\right]}
$$

En (3) la NSE se define como una métrica normalizada que ajusta los valores entre los datos observados y los simulados para medir la dispersión entre estos. Los valores posibles del NSE están en el rango de menos infinito hasta uno; cuando es igual a uno, indica una coincidencia perfecta entre las simulaciones y las observaciones, entre más cerca a uno esté el resultado, más preciso es el modelo.

$$
N S E=1-\left[\frac{\sum_{i=1}^{n}\left(Y_{i}^{o b s}-Y_{i}^{s i m}\right)^{2}}{\sum_{i=1}^{n}\left(Y_{i}^{\text {obs }}-Y_{i}^{\text {mean }}\right)^{2}}\right]
$$

Para todas las métricas, $n$ es el número total de datos de la serie de tiempo, $Y_{i}^{\text {obs }}$ es el valor en el tiempo $i$ de la variable observada, $Y_{i}^{\text {sim }}$ es el valor en el tiempo $i$ de la variable simulada y $Y_{i}^{\text {mean }}$ es el promedio de los datos observados. Para evaluar el desempeño de los MCR con base en las métricas seleccionadas se tomaron como referencia los rangos definidos por [55], quienes clasifican el desempeño como: Muy bueno *, Bueno **, Satisfactorio +, Insatisfactorio (Tabla 1). 
Tabla 1. Rangos para la evaluación del desempeño de los MCR según las métricas RSR, NSE y PBIAS Fuente: Adaptado de [56].

\begin{tabular}{llll}
\hline & & \multicolumn{1}{c}{ Métricas } & \\
\hline Desempeño & RSR & NSE & PBIAS (\%) \\
Muy bueno* $^{*}$ & $0.00 \leq \mathrm{RSR} \leq 0.50$ & $0.75<\mathrm{NSE} \leq 1.00$ & PBIAS $< \pm 10$ \\
Bueno** & $0.50<\mathrm{RSR} \leq 0.60$ & $0.65<\mathrm{NSE} \leq 0.75$ & $\pm 10 \leq \mathrm{PBIAS}< \pm 15$ \\
Satisfactorio+ $^{*}$ & $0.60<\mathrm{RSR} \leq 0.70$ & $0.50<\mathrm{NSE} \leq 0.65$ & $\pm 15 \leq$ PBIAS $< \pm 25$ \\
Insatisfactorio $^{\circ}$ & $\mathrm{RSR}>0.70$ & $\mathrm{NSE} \leq 0.50$ & $\mathrm{PBIAS} \geq \pm 25$ \\
\hline
\end{tabular}

En (4) se tomará la ecuación de balance hidrológico de largo plazo [56] como referencia para estimar la representación de los caudales en la cuenca.

$$
\mathrm{P}-\mathrm{E}=\mathrm{R}
$$

Donde $P$ representa la precipitación promedio de largo plazo, $E$ la evaporación total promedio de largo plazo y R la estimación indirecta de la escorrentía promedio de largo plazo. Se estimará el valor de $\mathrm{R}$ con base en las simulaciones de los MCR y la información de referencia para precipitación y evaporación total. Es importante aclarar que la información sobre la variable E que ofrecen los MCR del experimento CORDEX-CORE corresponde a la evapotranspiración real [57]. Finalmente, esta escorrentía será comparada con los caudales reportados en la estación Aguaverde del IDEAM y los datos de escorrentía reportados por el reanálisis ERA5. Por su utilidad para la representación del balance de agua superficial en una cuenca, (4) se ha empleado en diferentes estudios para evaluar el desempeño de fuentes de información [58]-[61].

\section{RESULTADOS Y DISCUSIÓN}

La Figura 2 muestra la distribución espacial de la precipitación promedio multianual según las estaciones de IDEAM en la cuenca del río Meta. Se evidenció que la precipitación promedio mensual varía en la zona de estudio en un rango de $67 \mathrm{~mm} / \mathrm{mes}$ a aproximadamente $600 \mathrm{~mm} / \mathrm{mes}$, donde los valores por encima de $250 \mathrm{~mm} / \mathrm{mes}$ se encuentran en la zona del Piedemonte de la cordillera Oriental y los valores más bajos $(67-100 \mathrm{~mm} / \mathrm{mes})$ en la zona del altiplano en los departamentos de Boyacá y Cundinamarca.

Particularmente, sobre el Piedemonte de la cordillera Oriental de los Andes se concentran más del $80 \%$ de las estaciones pluviométricas disponibles (69 estaciones), lo que resulta en una estación pluviométrica cada $412 \mathrm{~km}^{2}$, mientras menos del $20 \%$ (16 estaciones) se encuentran en el resto del territorio de estudio, una estación pluviométrica por cada $3100 \mathrm{~km}^{2}$.

Por otra parte, en la Figura 3 se muestran los campos de precipitación promedio mensual de los MCR con las condiciones de borde de tres los MCG: NorESM1-M, MPI-ESM-LR y HadGEM2. En general, se evidencia heterogeneidad en los valores promedio mensuales que arroja cada MCR. Por ejemplo, RegCM-Had exhibe alta precipitación (350-400 mm/mes) en el sur de la cuenca de estudio, mientras RegCM-MPI muestra alta precipitación en el norte. 


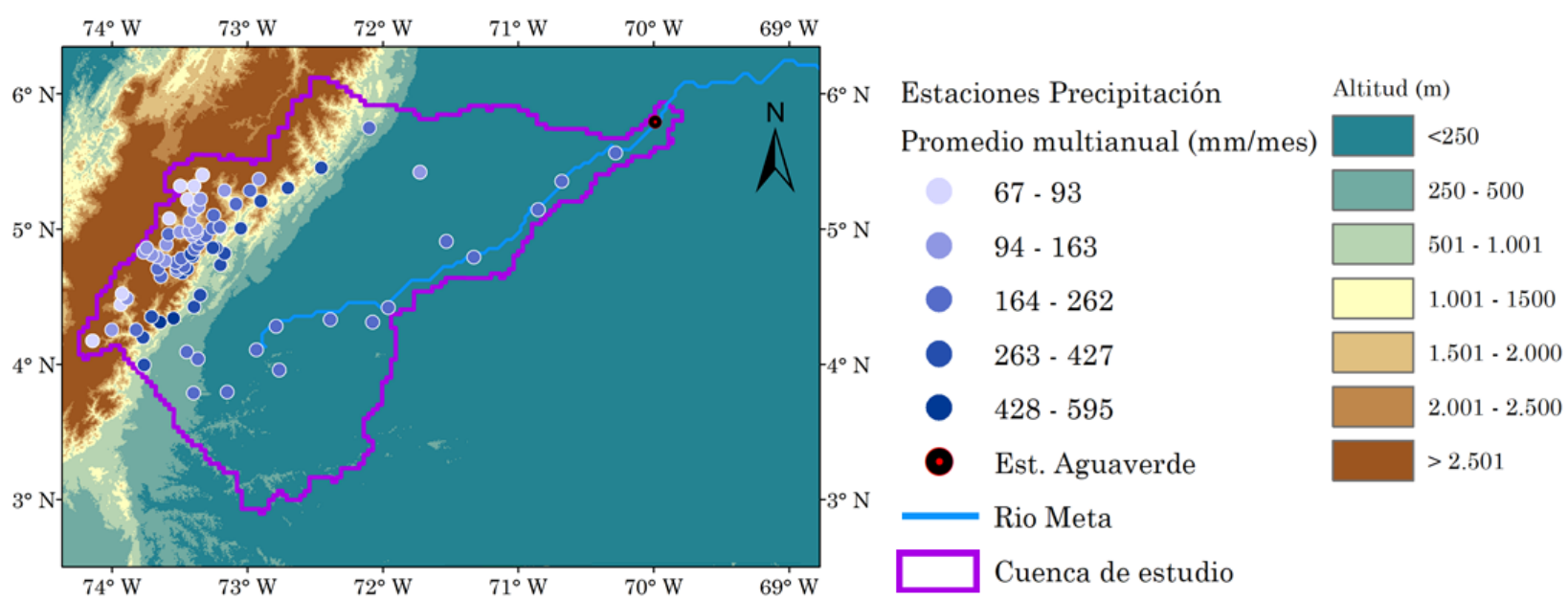

Figura 2. Corte sobre la cuenca del río Meta para el análisis de CORDEX y localización de las estaciones de precipitación y caudal del IDEAM. Fuente: elaboración propia.
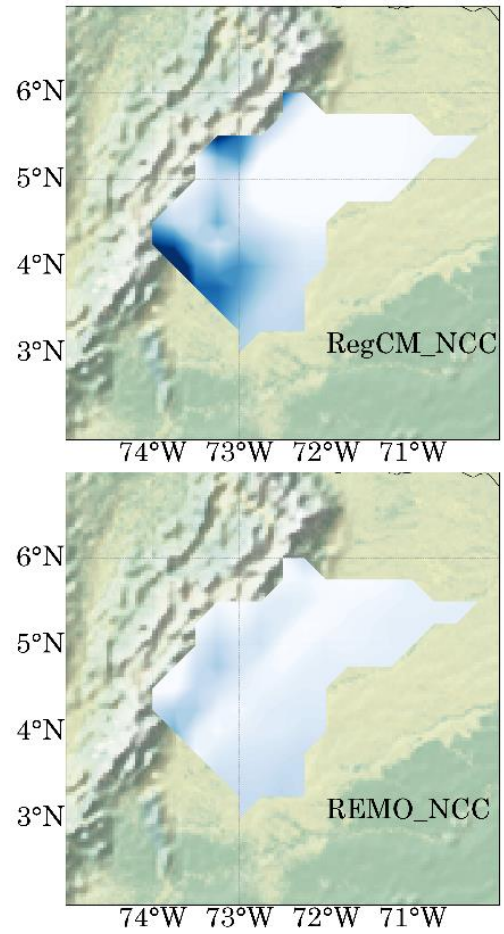
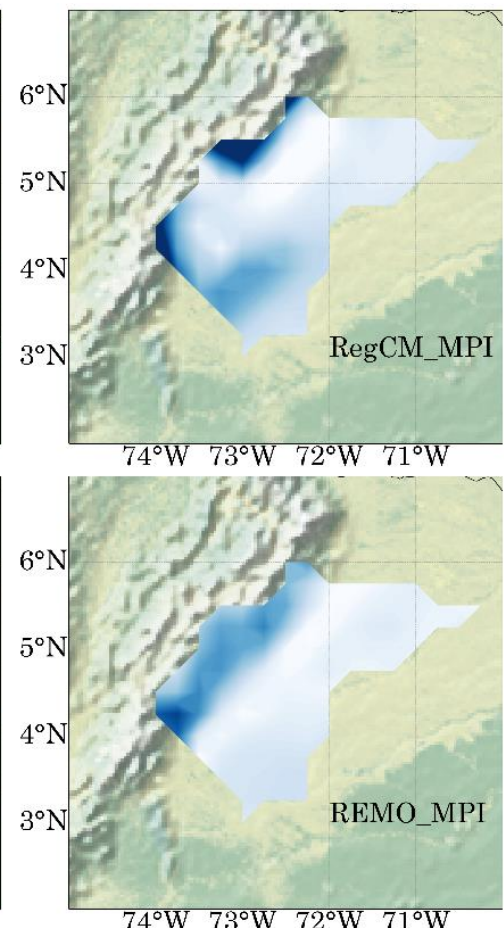

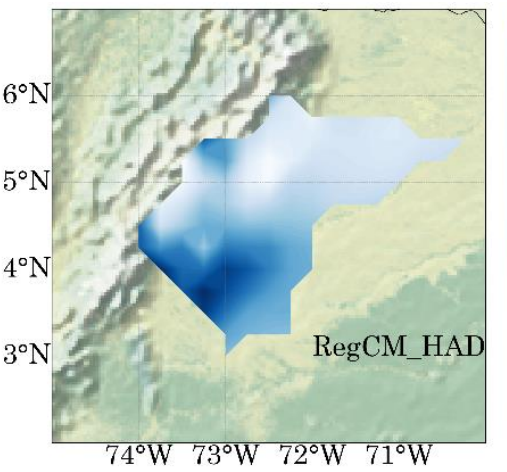

390

360

330

$300 \frac{\pi}{6}$

270 घ

240 로

$210^{\text {근 }}$

$-180$

$-150$

120

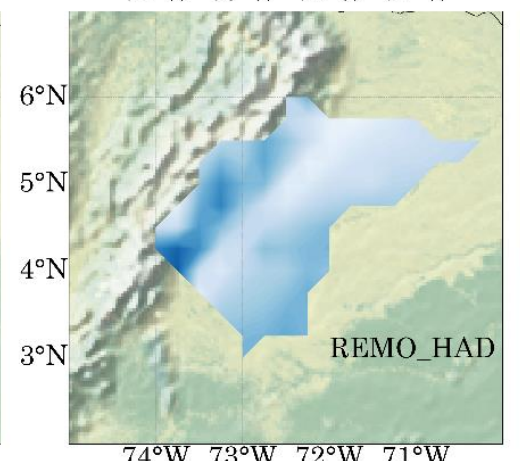

390

360

330

$300 \frac{n}{0}$

270

240 르

$210^{\text {근 }}$

180

$-150$

$-120$

MCR y sus respectivos GCM forzantes. Fuente: elaboración propia.

De igual manera, REMO-MPI muestra precipitación alta en el Piedemonte (200$400 \mathrm{~mm} / \mathrm{mes}$ ), mientras REMO-NOR sugiere una precipitación promedio mensual casi homogénea en toda la cuenca de interés (100-200 mm/mes). Por lo tanto, la heterogeneidad que presentan los resultados particulares de los MCR en la estimación de la precipitación media mensual pone de manifiesto la importancia de utilizar ensambles para minimizar la incertidumbre en la selección de una realización en particular [53].

La Figura 4 muestra los ciclos anuales de precipitación y evaporación total sobre la zona de estudio, para las fuentes de referencia y los ensambles de MCR en el periodo 1986-2005. 

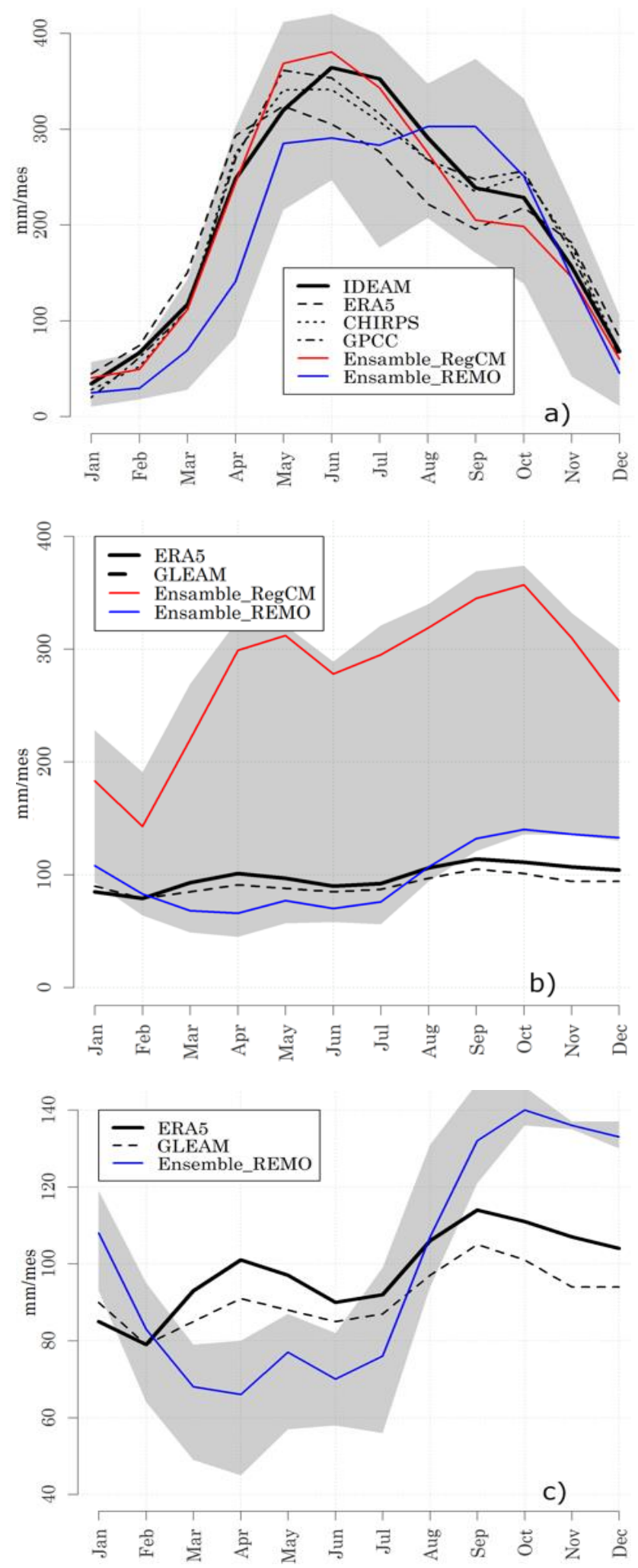

Figura 4. Ciclo anual de la precipitación y la evaporación total. (a) Ciclo anual de la precipitación según los datos de IDEAM, CHIRPS, ERA5, GPCC y ensambles RegCM y REMO. (b) Ciclo anual de la evaporación total según los datos de ERA5, GLEAM y ensambles RegCM y REMO. (c) Evaporación total según el ensamble REMO con relación a las fuentes ERA5 y GLEAM. El sombreado gris representa el área comprendida entre los valores máximos y mínimos mensuales de las corridas de los ensambles de MCR. Fuente: elaboración propia. 
La Figura 4-a evidencia que la mayoría de las fuentes de información de precipitación exhiben un ciclo anual con distribución unimodal. Es decir, sólo se presenta un mínimo y un máximo de precipitación mensual durante el año. Se evidenció una temporada de lluvia entre abril y noviembre, con precipitación máxima de $364 \mathrm{~mm}$ en el mes de junio. También una temporada seca entre diciembre y marzo, con precipitación mínima de $34.28 \mathrm{~mm}$ en el mes de enero, lo cual coincide con la temporada de actividad del Chorro del Orinoco y la ausencia de la ZCIT [6].

De los modelos de CORDEX-CORE, el ensamble del modelo REMO (línea azul) presenta los valores de precipitación más alejados de las observaciones, mostrando subestimación para el periodo noviembre-agosto con relación a los valores registrados por las estaciones del IDEAM (línea negra continua). También se muestra que el ensamble del modelo RegCM (línea roja) presenta valores promedio mensuales multianuales muy cercanos al promedio de las estaciones del IDEAM durante los meses de noviembre hasta abril. Sin embargo, durante los meses de mayo hasta junio dicho modelo sobrestima las observaciones, mientras que durante julio-noviembre, las subestima. Es de resaltar que ni REMO ni las fuentes satelitales logran representar de manera adecuada el máximo de precipitación reportado en el mes de junio por los datos del IDEAM. No obstante, no hay gran dispersión entre los valores observados por el IDEAM, los valores del ensamble del modelo RegCM y las demás fuentes de referencia.

La Figura 4-b muestra el ciclo anual de evaporación total para el mismo periodo de análisis sobre la cuenca de estudio, los resultados probaron que el ensamble del modelo RegCM difiere significativamente en orden de magnitud de los valores reportados por ERA5, GLEAM y el ensamble de las corridas del modelo REMO. En la Figura 4-c se presenta un acercamiento a la comparación gráfica entre el ensamble REMO y las fuentes ERA5 y GLEAM. Mientras ERA5 y GLEAM presentan variabilidad bimodal y evaporación total mensual promedio entre 80 y $100 \mathrm{~mm} / \mathrm{mes}$ a lo largo del año, REMO sugiere un ciclo anual predominantemente unimodal con valores máximos alrededor de $140 \mathrm{~mm} / \mathrm{mes}$ durante septiembre-octubre, y valores mínimos cercanos a $70 \mathrm{~mm} / \mathrm{mes}$ durante abril-junio.

Lo que sugiere que ninguno de los dos ensambles hace una buena representación del ciclo anual de la evaporación total.

La Figura 5-a muestra los diagramas de cajas para la distribución de los valores mensuales de precipitación y evaporación total obtenidos de los ensambles de los MCR y las fuentes de referencia. Para la precipitación, se evidenció que todas las referencias estaban alrededor de $200 \mathrm{~mm} / \mathrm{mes}$, además las fuentes de información exhibieron mayor variabilidad en los valores asociados al primer cuartil (precipitación baja), mientras que los valores asociados al máximo del tercer cuartil (precipitación alta) presentaron similitud en orden de magnitud. También se demostró que la fuente que presenta valores promedio mensuales más cercanos a las observaciones es GPCC, teniendo un rango de variabilidad y una media similares a los del IDEAM. En cuanto a la evaporación total, la Figura 5-b muestra que las referencias ERA5 y GLEAM tienen valores alrededor de los $100 \mathrm{~mm} / \mathrm{mes}$, al igual que el ensamble del modelo REMO. Si bien REMO representa bien la mediana, presenta una mayor variabilidad en la distribución de la evaporación total con respecto a los valores de referencia. 
Precipitación

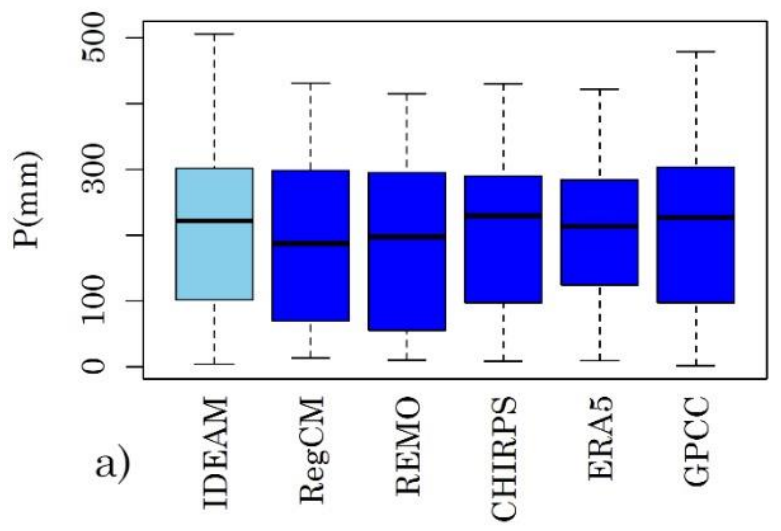

IDEAM/RegCM

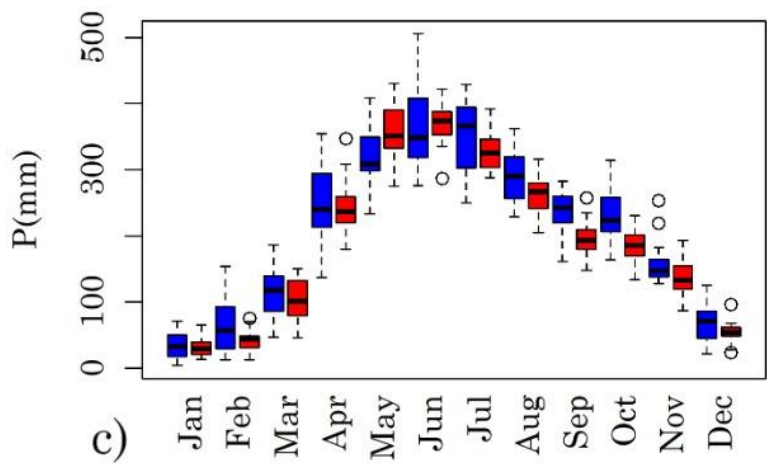

Evaporación total

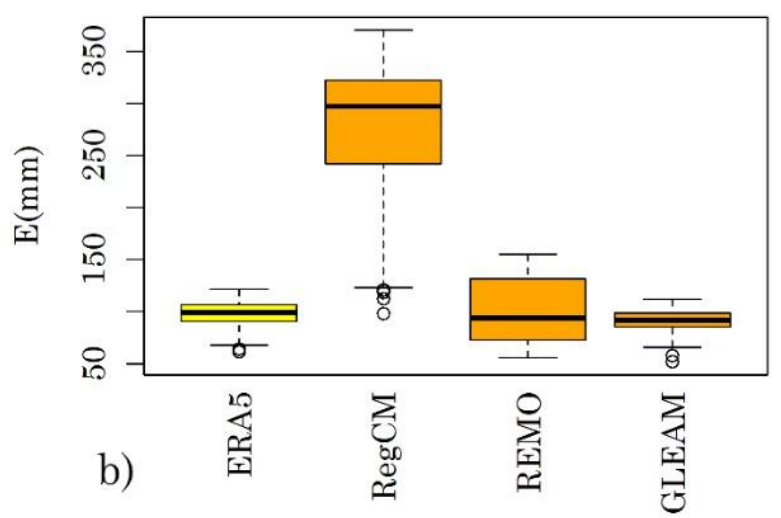

ERA5/REMO

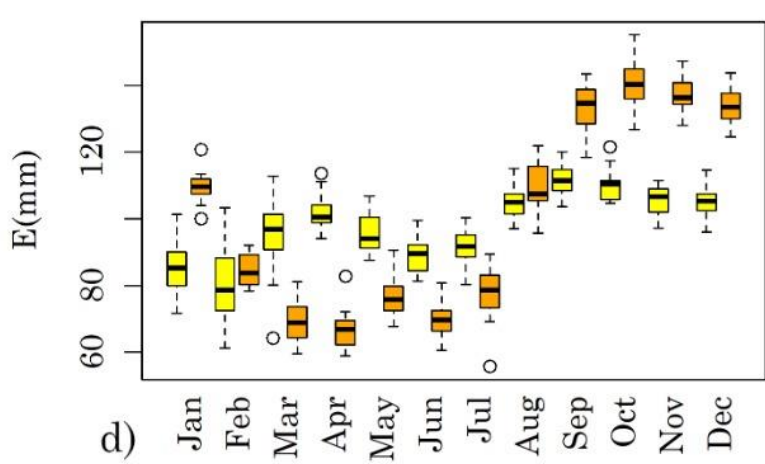

Figura 5. Diagrama de cajas para las series de tiempo de precipitación (a) y evaporación total (b) mensual promedio en la zona de estudio. (c) Diagramas de cajas para la precipitación mensual según los datos de IDEAM, CHIRPS, ERA5, GPCC y los ensambles de RegCM y REMO. (d) Diagramas de cajas para la evaporación total según los datos de ERA5, GLEAM y los ensambles de RegCM y REMO. Fuente: elaboración propia.

Además, se revisó la variabilidad de los valores mensuales de los ensambles con relación a las observaciones utilizando diagramas de cajas mes a mes para los ensambles que presentaron mayor similitud en el ciclo anual promedio. La Figura 5-c muestra que los valores mensuales del ensamble RegCM (rojo) presentan menor variabilidad que los de referencia (azul), dicha diferencia en la dispersión de los valores mensuales puede atribuirse a que los registros pluviométricos son puntuales y no tienen una distribución espacial uniforme, mientras que la información de RegCM proviene de datos interpolados espacialmente en una retícula uniforme. La Figura 5-d muestra que los valores mensuales del ensamble REMO (naranja) presentan diferencias en la estacionalidad de la evaporación total, pero ambas fuentes de datos coinciden en que el periodo septiembre-diciembre presenta valores altos de evaporación total mensual entre $100 \mathrm{~mm} / \mathrm{mes}$ y $140 \mathrm{~mm} / \mathrm{mes}$.

La Tabla 2 muestra los resultados de las métricas cuantitativas de rendimiento para la precipitación con relación a los datos IDEAM de los ensambles de MCR y otras fuentes de referencia. En general, se muestra que el ensamble de MCR que mejor simuló las observaciones fue RegCM. En este sentido, se puede resaltar que RegCM tiene una alta correlación $(R=0.91)$ con las observaciones obtenidas por el IDEAM, reflejando una 
importante asociación lineal entre las bases de datos. El coeficiente de determinación $\left(R^{2}=\right.$ 0.82) indica que el modelo RegCM representa más del $80 \%$ de la varianza de las observaciones. Mientras que el error típico es de $22.12 \mathrm{~mm}$ con relación al valor promedio mensual observado en los datos del IDEAM. Asimismo, el ensamble RegCM presenta una baja diferencia porcentual con un $3 \%$ de desviación con respecto al IDEAM. La clasificación del rendimiento para cada una de las métricas se basa en los rangos presentados en la Tabla 1.

Tabla 2. Métricas cuantitativas de rendimiento de los ensambles de los MCR y las fuentes observacionales de precipitación con relación a la información de IDEAM. Fuente: elaboración propia.

\begin{tabular}{|c|c|c|c|c|c|c|c|c|c|}
\hline \multicolumn{10}{|c|}{ Precipitación } \\
\hline Fuentes & $\mu(\mathrm{mm})$ & $\sigma(\mathrm{mm})$ & $\begin{array}{l}\text { Error } \\
\text { típico } \\
(\mathrm{mm})\end{array}$ & Dif. & $\mathrm{R}$ & $\mathrm{R} 2$ & RSR & NSE & PBIAS \\
\hline RegCM & 201.83 & 123.50 & 22.12 & $3 \%$ & 0.91 & 0.82 & $0.46^{*}$ & $0.79 *$ & $6.99 *$ \\
\hline REMO & 180.86 & 116.37 & 47.71 & $13 \%$ & 0.83 & 0.69 & $0.61 * *$ & $0.63+$ & $11.40^{* *}$ \\
\hline CHIRPS & 204.75 & 113.98 & 22.90 & $1 \%$ & 0.86 & 0.74 & $0.52^{* *}$ & $0.73^{* *}$ & $1.55^{*}$ \\
\hline ERA5 & 197.36 & 94.61 & 33.15 & $5 \%$ & 0.84 & 0.73 & $0.55^{* *}$ & $0.70 * *$ & $0.93^{*}$ \\
\hline GPCC & 208.17 & 119.28 & 23.36 & $-1 \%$ & 0.85 & 0.73 & $0.55^{* *}$ & $0.70^{* *}$ & $-0.51^{*}$ \\
\hline \multicolumn{10}{|c|}{ Evaporación total } \\
\hline Fuentes & $\mu(\mathrm{mm})$ & $\sigma(\mathrm{mm})$ & $\begin{array}{l}\text { Error } \\
\text { típico } \\
(\mathrm{mm})\end{array}$ & Dif. & $\mathrm{R}$ & $\mathrm{R} 2$ & RSR & NSE & PBIAS \\
\hline RegCM & 276.20 & 65.06 & 35.02 & $381 \%$ & 0.67 & 0.45 & $16.56^{\circ}$ & $-273.08^{\circ}$ & $-181.77^{\circ}$ \\
\hline REMO & 99.66 & 29.37 & 23.14 & $201 \%$ & 0.52 & 0.27 & $2.23^{\circ}$ & $-3.95^{\circ}$ & $-2.69 *$ \\
\hline GLEAM & 91.47 & 7.40 & 3.14 & $193 \%$ & 0.77 & 0.59 & $0.86^{\circ}$ & $0.26^{\circ}$ & $6.65^{*}$ \\
\hline
\end{tabular}

Se siguió evidenciando que el modelo REMO es el que presenta mayor diferencia con relación a los valores de referencia de precipitación, este reporta una diferencia porcentual de $13 \%$ por encima del valor de las observaciones IDEAM. También, se observó un error típico de $47.71 \mathrm{~mm}$ con relación a la media de las observaciones. Por su parte, para el ensamble REMO, métricas como el coeficiente de correlación $(R=0.83)$ y el coeficiente de determinación $\left(R^{2}=0.69\right)$ presentan una reducción significativa en la representación de las observaciones comparado con el ensamble RegCM. Además, el PBIAS 12.26 indica un aumento en el sesgo de REMO con relación a las observaciones IDEAM, mientras las métricas RSR y NSE cercanas a 0.60 reflejan un rendimiento satisfactorio pero inferior al del ensamble RegCM.

Por otra parte, para la evaporación total, se tomaron los datos de ERA5 como referencia y se evaluó el desempeño de los ensambles de los MCR, así como de los datos GLEAM. La Tabla 1 evidencia que el ensamble REMO presenta valores de evaporación total menos alejados de los reportados por ERA5. Sin embargo, ni REMO ni RegCM exhiben métricas de rendimiento satisfactorias para la evaporación total con relación a los datos de ERA5.

Para la estimación de caudales se utilizó la ecuación de balance hídrico de largo plazo (4) considerando la información de precipitación del ensamble con el mejor desempeño (RegCM), evaporación total del ensamble con el mejor desempeño (REMO), la evaporación total reportada por fuentes de referencia (GLEAM y ERA5) y la precipitación según las estaciones de IDEAM. Mientras que el caudal promedio multianual observado en la estación Aguaverde del IDEAM (código 35267080) es de $3346.28 \mathrm{~m}^{3} / \mathrm{s}$, las estimaciones desarrolladas utilizando 
las bases de datos seleccionadas llevan a los siguientes resultados en magnitud y en porcentaje con respecto a las observaciones: IDEAM-Ens_REMO: $3237.83 \mathrm{~m}^{3 / \mathrm{s}}(97 \%)$; IDEAM-GLEAM: 3484.52 m³/s (104\%); IDEAM-E_ERA5: $3276.85 \mathrm{~m}^{3} / \mathrm{s}$ (98 \%); Ens_RegCMEns_REMO: $3078.62 \mathrm{~m}^{3} / \mathrm{s}$ (92 \%). En general, puede concluirse que tanto la estimación de caudal empleando la evaporación total del ensamble REMO y la información del IDEAM, la estimación de caudal usando evaporación total de GLEAM y ERA5, y la estimación del caudal empleando los dos mejores ensambles de MCR exhiben errores porcentuales inferiores al $5.0 \%$ en la estimación del caudal promedio multianual.

No obstante, múltiples aplicaciones hidrológicas (Ej.: planeación del recurso hídrico, actividades agrícolas, planeación energética, entre otras) requieren de la adecuada caracterización y estimación del ciclo anual. Con este fin, se verificó si la información de los MCR permite hacer una adecuada estimación del caudal promedio mensual multianual. Al respecto, encontramos que al emplear la información de precipitación y/o evaporación total de los MCR no es posible hacer una estimación adecuada ni de la temporalidad del ciclo anual de los caudales ni de su magnitud promedio mensual. La Figura 6 muestra que los registros de caudal de IDEAM y las estimaciones exhiben un ciclo anual de caudales unimodal, pero estos ciclos difieren en las temporadas de caudales máximos y mínimos. Además, todos los MCR exhiben valores de evaporación total mensual superiores a la precipitación en el periodo octubre-marzo sugiriendo un déficit hídrico en la cuenca que difiere significativamente de las observaciones. Por su parte, durante la temporada abril-julio los caudales estimados utilizando información de los MCR arrojan valores superiores a los registrados por IDEAM, lo que sugiere que durante dicho periodo los MCR subestiman los valores de evaporación total que se presentan en la zona.

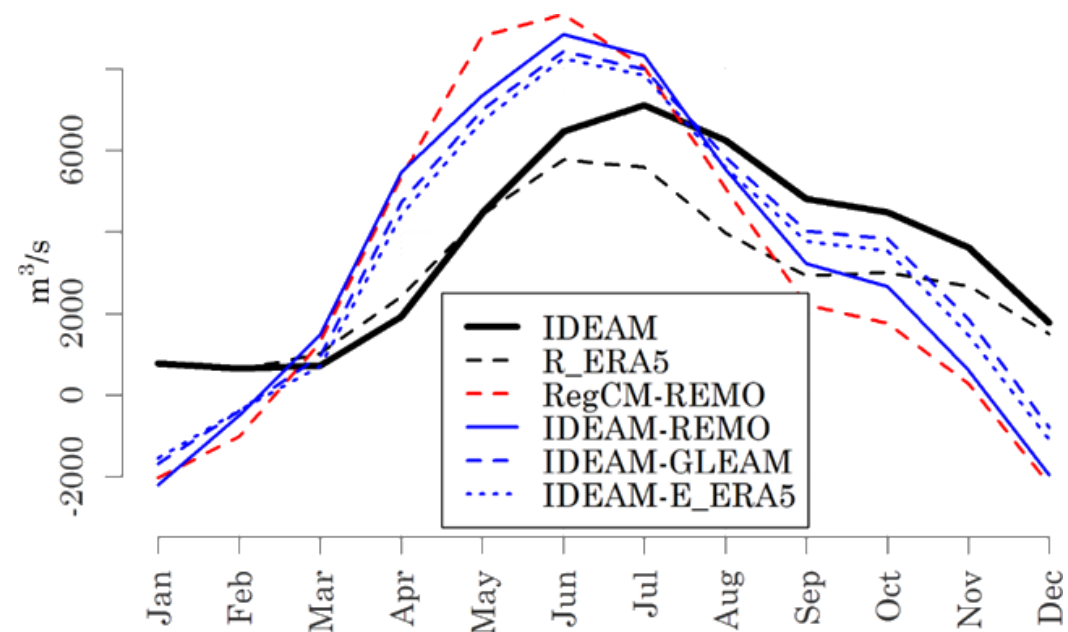

Figura 6. Ciclo anual de los caudales promedio mensuales en la cuenca hidrográfica aferente a la estación Aguaverde (código 35267080) del IDEAM. Las líneas negras representan la información de referencia del IDEAM (continua) y de escorrentía superficial de ERA5 (discontinua). Fuente: elaboración propia.

\section{CONCLUSIONES}

Gráficamente se identificó que el MCR que más se aproxima a las observaciones de precipitación obtenidas por el IDEAM para el Orinoco colombiano es RegCM. Sin embargo, cabe resaltar que las fuentes interpoladas satelitales también muestran valores similares a los valores obtenidos por el IDEAM para la zona de estudio y la escala temporal utilizada. 
El análisis comparativo a través de métricas de rendimiento permite concluir y verificar que RegCM es el MCR que mejor representa el ciclo unimodal de la precipitación en la cuenca de estudio. En relación con esto, aunque RegCM es el modelo que muestra valores más aproximados a los del IDEAM, en algunas épocas del año, especialmente en mayo, subestima en gran medida las observaciones. Por otra parte, también se verificó que la precipitación según REMO presenta un mayor sesgo y dispersión que RegCM, lo cual nos permite concluir que tanto el ensamble de RegCM como las CHIRPS, GPCC y ERA5 podrían ser empleados como fuentes para el estudio de la precipitación en la zona de estudio. No obstante, la precipitación mensual del MCR REMO presenta resultados satisfactorios para la representación del ciclo anual promedio y podría ser utilizado para describir el ciclo anual de la precipitación en cuencas sin instrumentación.

Gráficamente es posible hacer una primera inspección de los resultados de evaporación total y determinar que ninguno de los MCR representa adecuadamente dicha variable. Una vez calculadas las métricas de desempeño, se encuentra que ninguna de las fuentes comparadas con ERA5 logra representar de forma satisfactoria la evaporación total. Estos resultados confirman las dificultades para la consecución de información de buena calidad de esta variable, de la cual no se cuentan redes consolidadas de medición en el país, por lo cual se recomienda para aplicaciones hidrológicas hacer una estimación indirecta de dicha variable a partir de la información de temperatura disponible.

Los ensambles de los MCR son una buena aproximación para la adecuada representación de la precipitación. Los resultados aquí expuestos confirman que el ensamble de RegCM representa adecuadamente la precipitación en la zona de estudio, esto podría estar relacionado con las diferentes parametrizaciones por dominio del modelo RegCM [25], [26], abriendo la puerta hacia una mayor confiabilidad de los estudios de impacto de cambio climático basados en la regionalización de los resultados de MCG. Una mayor confiabilidad en la representación de la precipitación por parte de los MCR incrementa las opciones de información hidrológica para quienes desarrollan actividades en temas de recursos hídricos en cuencas poco instrumentadas.

El análisis comparativo utilizando la ecuación de balance hídrico de largo plazo permite concluir que los datos de precipitación del ensamble de RegCM y los datos de evaporación total del ensamble REMO permiten una estimación adecuada del caudal promedio anual de largo plazo. Sin embargo, ni los datos de evaporación total reportados por REMO y RegCM, ni los reportados por las fuentes ERA5 y GLEAM, permiten hacer una estimación de la temporalidad y magnitud del ciclo anual de caudales en la cuenca de estudio. La selección de cada MCR dependerá del enfoque del estudio a desarrollar. Para estudios apoyados en el análisis del comportamiento temporal de las variables, será conveniente seleccionar el MCR que representa mejor el ciclo anual en la zona de estudio.

En general, los MCRs tienen múltiples fuentes de incertidumbre, tales como la conceptualización y parametrización de cada MCR, la incertidumbre en los escenarios de emisión para proyecciones futuras en el contexto de cambio climático, incertidumbre en los procesos de reducción de escala (downscaling), incertidumbre en la sensibilidad de los modelos y la incertidumbre asociada a la adecuada representación de la complejidad y nolinealidad de la dinámica del sistema climático terrestre[62], [64]. Además, también debe tenerse en cuenta la incertidumbre en la respuesta del sistema climático debido a la acción humana y el forzamiento radiativo como consecuencia de los cambios en concentraciones futuras de emisiones de gases de efecto invernadero [62]. Asimismo, los modelos que acoplan la dinámica atmosférica y oceánica muestran incertidumbre den el campo del desarrollo, aunque estos son ecuaciones que describen los distintos componentes a través de sistemas numéricos y parametrizaciones de procesos físicos, las simulaciones realizadas con distintos 
modelos muestran dispersión, reflejando así la sensibilidad del clima a pequeños cambios en el forzamiento, también se añaden a las incertidumbres los subsistemas de los modelos climáticos como el realismo en las variaciones, el modelado del suelo y usos de este [65]. A diferencia de los modelos de pronóstico del estado del tiempo, los modelos de simulación del clima buscan dar una representación de los patrones de largo plazo en las variables del sistema.

En particular, en el caso de este estudio, se encontró que los MCRs no hacen una adecuada representación de la evaporación total. Según la literatura, los problemas en la representación de esta variable son frecuentes debido a la dificultad para la parametrización de los procesos que configuran la evaporación total y a las pocas mediciones que se tienen en tierra para poder comprender la variable y validar las representaciones de las interacciones suelo atmósfera [65]-[68], la sensibilidad de la evaporación con respecto a los flujos de energía en la superficie [69], [70], las limitaciones en la representación de la convección, disponibilidad de humedad y en topografía en las regiones tropicales del planeta puede influir en la sobre o subestimación de la evaporación [71], [72].

Como trabajo futuro, se propone el estudio de la regionalización de variables hidrológicas a través de la caracterización de patrones espaciales y de escalamiento en cuencas no instrumentadas utilizando información de los MCR.

\section{AGRADECIMIENTOS}

CFV agradece al proyecto "Evaluación del desempeño y proyecciones para la representación de la disponibilidad de agua superficial de los modelos regionales en el dominio SAM-CORDEX para las cuencas del Río Amazonas y Del Plata” por su financiación durante el desarrollo de este proyecto. HDS agradece a Colciencias por la beca para doctorados nacionales convocatoria 617-2. Los autores también agradecen a las instituciones que permiten el acceso libre a sus bases de datos: Las simulaciones de MCR de CORDEX se encuentran disponibles gracias al Earth System Grid Federation en su página web (https://esgf.llnl.gov/), ERA5 está disponible en la página web del European Copernicus Programme (https://www.copernicus.eu/), CHIRPS se encuentra disponible gracias a la University of California Santa Barbara Climate Hazards Center (https://www.chc.ucsb.edu/data), GPCC está disponible gracias al Physical Sciences Laboratory (https://psl.noaa.gov/data/gridded/data.gpcc.html), GLEAM está disponible en la página web del proyecto (https://www.gleam.eu/) y la información observacional de estaciones en Colombia se encuentra disponible gracias al IDEAM (www.ideam.gov.co).

\section{CONFLICTO DE INTERESES}

Los autores declaran no tener conflictos de intereses.

\section{CONTRIBUCIÓN DE LOS AUTORES}

Carolina Florian Vergara se encargó de la obtención y procesamiento de los datos, preparación del manuscrito inicial y análisis de la información. Carolina Florian Vergara, Hernán D. Salas y Alejandro Builes-Jaramillo participaron en la conceptualización del estudio, en el análisis y discusión de resultados, escritura y edición del documento. 


\section{REFERENCIAS}

[1] S. Riveros-Angarita, "La Orinoquia Colombiana," Soc. Geogr. Colomb., vol. 36, no. 118, pp. 1-9, 2010. URL

[2] V. Urrea; A. Ochoa; O. Mesa, "Seasonality of Rainfall in Colombia," Water Resour. Res., vol. 55, no. 5, pp. 4149-4162, May. 2019. https://doi.org/10.1029/2018WR023316.

[3] G. Berry; M. J. Reeder, "Objective identification of the intertropical convergence Zone: and trends from the ERA-Interim,” J. Clim., vol. 27, no. 5, pp. 1894-1909, Mar. 2014. https://doi.org/10.1175/JCLI-D-1300339.1

[4] P. A. Arias; J. A. Martínez; J. D. Mejía; M. J. Pazos; J. C. Espinoza; S. Wongchuig-Correa, "Changes in Normalized Difference Vegetation Index in the Orinoco and Amazon River Basins: Links to Tropical Atlantic Surface Temperatures," J. Clim., vol. 33, no. 19, pp. 8537-8559, Oct. 2020. https://doi.org/10.1175/JCLI-D-19-0696.1

[5] G. Jiménez-Sánchez; P. M. Markowski; V. Jewtoukoff; G. S. Young; D. J. Stensrud, "The Orinoco LowLevel Jet: An Investigation of Its Characteristics and Evolution Using the WRF Model," Journal of Geophysical Research: Atmospheres, vol. 124, no. 20. pp. 10696-10711, 2019. https://doi.org/10.1029/2019JD030934

[6] G. Jiménez-Sánchez; P. M. Markowski; G. S. Young; D. J. Stensrud, "The Orinoco Low-Level Jet: An Investigation of its Mechanisms of Formation Using the WRF Model," J. Geophys. Res. Atmos., vol. 125, no. 13, pp. 1-23, 2020. https://doi.org/10.1029/2020jd032810

[7] F. Giorgi; C. Jones; G. Asrar, "Addressing climate information needs at the regional level: the CORDEX framework," Organ. Bull., vol. 50, no. 3, 2009. URL

[8] B. E. Oviedo Torres; G. Aristizábal León, "Guía de procedimiento para la generación de escenarios de cambio climático regional y local a partir de los modelos globales," Inst. Hidrol. , Meterología y Estud. Ambient., 2010. URL

[9] A. Rodríguez, "Evaluación de los modelos globales del clima utilizados para la generación de escenarios de cambio climático con el clima presente en Colombia,” Ideam-Meteo, 2010.

[10] P. Acevedo, "Comparación de series de precipitación con los GCM CCSM3, ECHAM5, HADGEM1 y MIROC 3.2 HIRES, para el siglo XX en Colombia," 2008.

[11] M. Rummukainen, "Added value in regional climate modeling," Wiley Interdiscip. Rev. Clim. Chang., vol. 7, no. 1, pp. 145-159, 2016. https://doi.org/10.1002/wcc.378

[12] K. E. Taylor; R. J. Stouffer; G. A. Meehl, "An overview of CMIP5 and the experiment design," Bull. Am. Meteorol. Soc., vol. 93, no. 4, pp. 485-498, 2012. https://doi.org/10.1175/BAMS-D-11-00094.1

[13] F. Giorgi, "Thirty Years of Regional Climate Modeling: Where Are We and Where Are We Going next?," J. Geophys. Res. Atmos., vol. 124, no. 11, pp. 5696-5723, 2019. https://doi.org/10.1029/2018JD030094

[14] T. Ambrizzi; M. S. Reboita; R. P. da Rocha; M. Llopart, "The state of the art and fundamental aspects of regional climate modeling in South America," Ann. N. Y. Acad. Sci., vol. 1436, no. 1, pp. 98-120, 2019, doi: https://doi.org/10.1111/nyas.13932

[15] A. V. Karmalkar; R. S. Bradley; H. F. Diaz, "Climate change in Central America and Mexico: Regional climate model validation and climate change projections," Clim. Dyn., vol. 37, no. 3, pp. 605-629, 2011. https://doi.org/10.1007/s00382-011-1099-9

[16] CORDEX, "CORDEX CORE Simulation Framework - Cordex,” 2020. URL

[17] D. Jacob et al., "A comprehensive model inter-comparison study investigating the water budget during the BALTEX-PIDCAP period," Meteorol. Atmos. Phys., vol. 77, no. 1-4, pp. 19-43, Sep. 2001. https://doi.org/10.1007/s007030170015

[18] F. Giorgi et al., "RegCM4: Model description and preliminary tests over multiple CORDEX domains," Clim. Res., vol. 52, no. 1, pp. 7-29, 2012. https://doi.org/10.3354/cr01018

[19] M. Ashfaq et al., "Robust late twenty-first century shift in the regional monsoons in RegCM-CORDEX simulations," Clim. Dyn., vol. 57, no. 5-6, pp. 1463-1488, Sep. 2021. https://doi.org/10.1007/s00382-02005306-2

[20] M. Llopart et al., "Assessing changes in the atmospheric water budget as drivers for precipitation change over two CORDEX-CORE domains," Clim. Dyn., vol. 57, no. 5-6, pp. 1615-1628, Sep. 2021. https://doi.org/10.1007/s00382-020-05539-1

[21] M. Falco; A. F. Carril; L. Z. X. Li; C. Cabrelli; C. G. Menéndez, "The potential added value of Regional Climate Models in South America using a multiresolution approach," Clim. Dyn., vol. 54, no. 3-4, pp. 15531569, Feb. 2020. https://doi.org/10.1007/s00382-019-05073-9

[22] A. Builes-Jaramillo;V. Pántano, "Comparison of spatial and temporal performance of two Regional Climate Models in the Amazon and La Plata river basins," Atmos. Res., vol. 250, no. November 2020, Mar. 2021. https://doi.org/10.1016/j.atmosres.2020.105413 
[23] M. G. R. Fahad; A. K. M. Saiful Islam; R. Nazari; M. Alfi Hasan; G. M. Tarekul Islam; S. K. Bala, "Regional changes of precipitation and temperature over Bangladesh using bias-corrected multi-model ensemble projections considering high-emission pathways," Int. J. Climatol., vol. 38, no. 4, pp. 1634-1648, Mar. 2018. https://doi.org/10.1002/joc.5284

[24] S. A. Solman; J. Blázquez, "Multiscale precipitation variability over South America: Analysis of the added value of CORDEX RCM simulations," Clim. Dyn., vol. 53, no. 3-4, pp. 1547-1565, Aug. 2019. https://doi.org/10.1007/s00382-019-04689-1

[25] G. Pang; X. Wang; D. Chen; M. Yang; L. Liu, "Evaluation of a climate simulation over the Yellow River Basin based on a regional climate model (REMO) within the CORDEX," Atmos. Res., vol. 254, p. 105522, Jun. 2021. https://doi.org/10.1016/j.atmosres.2021.105522

[26] F. Giorgi; E. Coppola; C. Teichmann; D. Jacob, "Editorial for the CORDEX-CORE Experiment I Special Issue," Clim. Dyn., vol. 57, no. 5-6, pp. 1265-1268, Sep. 2021. https://doi.org/10.1007/s00382-021-05902-w

[27] M. Bentsen et al., "The Norwegian Earth System Model, NorESM1-M - Part 1: Description and basic evaluation of the physical climate," Geosci. Model Dev., vol. 6, no. 3, pp. 687-720, 2013. https://doi.org/10.5194/gmd-6-687-2013

[28] M. A. Giorgetta et al., "Climate and carbon cycle changes from 1850 to 2100 in MPI-ESM simulations for the Coupled Model Intercomparison Project phase 5,” J. Adv. Model. Earth Syst., vol. 5, no. 3, pp. 572-597, 2013. https://doi.org/10.1002/jame.20038

[29] G. M. Martin et al., "The HadGEM2 family of Met Office Unified Model climate configurations," Geosci. Model Dev., vol. 4, no. 3, pp. 723-757, 2011.https://doi.org/10.5194/gmd-4-723-2011

[30] A. Allam; R. Moussa; W. Najem; C. Bocquillon, "Specific climate classification for Mediterranean hydrology and future evolution under Med-CORDEX regional climate model scenarios," Hydrol. Earth Syst. Sci., vol. 24, no. 9, pp. 4503-4521, Sep. 2020. https://doi.org/10.5194/hess-24-4503-2020

[31] R. Laprise et al., "Challenging some tenets of Regional Climate Modelling," Meteorol. Atmos. Phys., vol. 100, no. 1-4, pp. 3-22, Aug. 2008. https://doi.org/10.1007/s00703-008-0292-9

[32] A. Alexandru; R. de Elia; R. Laprise, "Internal variability in regional climate downscaling at the seasonal scale," Mon. Weather Rev., vol. 135, no. 9, pp. 3221-3238, Sep. 2007. https://doi.org/10.1175/MWR3456.1

[33] C. Teutschbein; J. Seibert, "Bias correction of regional climate model simulations for hydrological climatechange impact studies: Review and evaluation of different methods," J. Hydrol., vol. 456-457, pp. 12-29, Aug. 2012. https://doi.org/10.1016/j.jhydrol.2012.05.052

[34] F. das N. Roque da Silva; J. L. Drummond Alves; M. Cataldi, "Climate downscaling over South America for 1971-2000: application in SMAP rainfall-runoff model for Grande River Basin," Clim. Dyn., vol. 52, no. 1-2, pp. 681-696, 2019. https://doi.org/10.1007/s00382-018-4166-7

[35] D. Rosbjerg et al., Prediction of floods in ungauged basins. 2013.

[36] M. Sivapalan, "Prediction in ungauged basins: a grand challenge for theoretical hydrology," Hydrol. Process., vol. 17, no. 15, pp. 3163-3170, Oct. 2003. https://doi.org/10.1002/hyp.5155

[37] M. Hrachowitz et al., "A decade of Predictions in Ungauged Basins (PUB)-a review," Hydrol. Sci. J., vol. 58, no. 6, pp. 1198-1255, Jun. 2013. https://doi.org/10.1080/02626667.2013.803183

[38] Z. H. Xie et al., "Coupled modeling of land hydrology-regional climate including human carbon emission and water exploitation," Adv. Clim. Chang. Res., vol. 8, no. 2, pp. 68-79, Jun. 2017. https://doi.org/10.1016/j.accre.2017.05.001

[39] C. Teutschbein; J. Seibert, "Regional Climate Models for Hydrological Impact Studies at the Catchment Scale: A Review of Recent Modeling Strategies," Geogr. Compass, vol. 4, no. 7, pp. 834-860, Jul. 2010. https://doi.org/10.1111/j.1749-8198.2010.00357.x

[40] L. P. Graham; S. Hagemann; S. Jaun; M. Beniston, "On interpreting hydrological change from regional climate models," Clim. Change, vol. 81, no. SUPPL. 1, pp. 97-122, Mar. 2007. https://doi.org/10.1007/s10584-006-9217-0

[41] M. Velásquez Restrepo; G. Poveda Jaramillo, "Estimación del balance hídrico de la región Pacífica Colombiana,” Dyna, vol. 86, no. 208, pp. 297-306, 2019. https://doi.org/10.15446/dyna.v86n208.73587

[42] D. Mena Rentería; E. M. Espinosa; P. C. Soler; M. Cañón Ramos; F. S. Duarte; J. R. Palacios González, "Water supply failure probability under influence of climate change-Balsillas river basin case study," Rev. Fac. Ing. Univ. Antioquia, no. 103, Oct. 2020. https://doi.org/10.17533/udea.redin.20201008

[43] D. Mena; A. Solera; L. Restrepo; M. Pimiento; M. Cañón; F. Duarte, "An analysis of unmet water demand under climate change scenarios in the Gualí River Basin, Colombia, through the implementation of HydroBID and WEAP hydrological modeling tools," J. Water Clim. Chang., pp. 1-12, Feb. 2021. https://doi.org/10.2166/wcc.2019.118

[44] J. P. Pietikäinen et al., "The regional climate model REMO (v2015) coupled with the 1-D freshwater lake model FLake (v1): Fenno-Scandinavian climate and lakes," Geosci. Model Dev., vol. 11, no. 4, pp. 13211342, Aug. 2018. https://doi.org/10.5194/gmd-11-1321-2018 
[45] J. H. Jungclaus et al., "Characteristics of the ocean simulations in the Max Planck Institute Ocean Model (MPIOM) the ocean component of the MPI-Earth system model," J. Adv. Model. Earth Syst., vol. 5, no. 2, pp. 422-446, Jun. 2013. https://doi.org/10.1002/jame.20023

[46] D. Machiwal; M. K. Jha, "Time series analysis of hydrologic data for water resources planning and management: a review," J. Hydrol. Hydromechanics, vol. 54, no. 3, pp. 237-257, 2006. URL

[47] A. Aieb; K. Madani; M. Scarpa; B. Bonaccorso; K. Lefsih, "A new approach for processing climate missing databases applied to daily rainfall data in Soummam watershed, Algeria," Heliyon, vol. 5, no. 2, e01247, Feb. 2019. https://doi.org/10.1016/j.heliyon.2019.e01247

[48] S. M. Shaharudin et al., "Imputation methods for addressing missing data of monthly rainfall in Yogyakarta, Indonesia," Int. J. Adv. Trends Comput. Sci. Eng., vol. 9, no. 1.4, pp. 646-651, Sep. 2020. https://doi.org/10.30534/ijatcse/2020/9091.42020

[49] C. Funk et al., "The climate hazards infrared precipitation with stations - A new environmental record for monitoring extremes," Sci. Data, vol. 2, pp. 1-21, Dec. 2015. https://doi.org/10.1038/sdata.2015.66.

[50] H. Hersbach et al., "The ERA5 global reanalysis," Q. J. R. Meteorol. Soc., vol. 146, no. 730, pp. 1999-2049, Jul. 2020. https://doi.org/10.1002/qj.3803

[51] U. Schneider; A. Becker; P. Finger; A. Meyer-Christoffer; M. Ziese; B. Rudolf, "GPCC's new land surface precipitation climatology based on quality-controlled in situ data and its role in quantifying the global water cycle," Theor. Appl. Climatol., vol. 115, no. 1-2, pp. 15-40, Mar. 2014. https://doi.org/10.1007/s00704013-0860-x

[52] B. Martens et al., "GLEAM v3: Satellite-based land evaporation and root-zone soil moisture," Geosci. Model Dev., vol. 10, no. 5, pp. 1903-1925, May. 2017. https://doi.org/10.5194/gmd-10-1903-2017

[53] R. Hagedorn; F. J. Doblas-Reyes; T. N. Palmer, "The rationale behind the success of multi-model ensembles in seasonal forecasting - I. Basic concept," Tellus, Series A: Dynamic Meteorology and Oceanography, vol. 57, no. 3. pp. 219-233, 2005. https://doi.org/10.1111/j.1600-0870.2005.00103.x

[54] A. C. Palladino, "Gráfico de caja," Atención primaria salud, Epidemiol. e Inform. II, pp. 7-10, 2011. URL

[55] D. N. Moriasi; J. G. Arnold; M. W. Van Liew; R. L. Bingner; R. D. Harmel; T. L. Veith, "Model Evaluation Guidelines for Systematic Quantification of Accuracy in Watershed Simulations," Trans. ASABE, vol. 50, no. 3, pp. 885-900, 2007. https://doi.org/10.13031/2013.23153

[56] J. Peixoto; A. Oort, Physics of Climate. AIP-Press, 1993.

[57] WRCP, "CORDEX CORE Simulation Framework," 2018. URL

[58] A. Builes-Jaramillo; G. Poveda, "Conjoint Analysis of Surface and Atmospheric Water Balances in the Andes-Amazon System,” Water Resour. Res., vol. 54, no. 5, pp. 3472-3489, May 2018. https://doi.org/10.1029/2017WR021338

[59] L. Zhang; N. Potter; K. Hickel; Y. Zhang; Q. Shao, "Water balance modeling over variable time scales based on the Budyko framework - Model development and testing," J. Hydrol., vol. 360, no. 1-4, pp. 117-131, Oct. 2008. https://doi.org/10.1016/j.jhydrol.2008.07.021

[60] J. A. Marengo, "Characteristics and spatio-temporal variability of the Amazon River Basin Water Budget," Clim. Dyn., vol. 24, no. 1, pp. 11-22, Jan. 2005. https://doi.org/10.1007/s00382-004-0461-6

[61] M. Llopart; M. Simões Reboita; R. Porfírio da Rocha, "Assessment of multi-model climate projections of water resources over South America CORDEX domain," Clim. Dyn., vol. 54, no. 1-2, pp. 99-116, 2020. https://doi.org/10.1007/s00382-019-04990-z

[62] A. M. Foley, "Uncertainty in regional climate modelling: A review," Prog. Phys. Geogr. Earth Environ., vol. 34, no. 5, pp. 647-670, Oct. 2010. https://doi.org/10.1177/0309133310375654

[63] E. Hawkins; R. Sutton, "The Potential to Narrow Uncertainty in Regional Climate Predictions," Bull. Am. Meteorol. Soc., vol. 90, no. 8, pp. 1095-1108, Aug. 2009. https://doi.org/10.1175/2009BAMS2607.1

[64] J. Gutiérrez; M. Pons, "Modelización numérica del cambio climático: bases científicas, incertidumbres y proyecciones para la Península Ibérica," Cuaternario y Geomorfol., vol. 20, no. 3, pp. 15-28, 2006. URL

[65] Y. Shi; M. Yu; A. Erfanian; G. Wang, "Modeling the Dynamic Vegetation-Climate System over China Using a Coupled Regional Model," J. Clim., vol. 31, no. 15, pp. 6027-6049, Aug. 2018. https://doi.org/10.1175/JCLID-17-0191.1

[66] A. M. Bryan; A. L. Steiner; D. J. Posselt, "Regional modeling of surface-atmosphere interactions and their impact on Great Lakes hydroclimate," J. Geophys. Res. Atmos., vol. 120, no. 3, pp. 1044-1064, Feb. 2015. https://doi.org/10.1002/2014JD022316

[67] J. C. A. Baker et al., "Evapotranspiration in the Amazon: spatial patterns, seasonality, and recent trends in observations, reanalysis, and climate models," Hydrol. Earth Syst. Sci., vol. 25, no. 4, pp. 2279-2300, Apr. 2021. https://doi.org/10.5194/hess-25-2279-2021

[68] J. Cuxart; A. Verhoef; T. Marthews; J. Evans, "Current Challenges in Evapotranspiration Determination, GEWEX News,” 2021. URL 
[69] A. A. Sörensson; R. C. Ruscica, "Intercomparison and Uncertainty Assessment of Nine Evapotranspiration Estimates Over South America," Water Resour. Res., vol. 54, no. 4, pp. 2891-2908, Apr. 2018. https://doi.org/10.1002/2017WR021682

[70] P. C. D. Milly; K. A. Dunne, "On the Hydrologic Adjustment of Climate-Model Projections: The Potential Pitfall of Potential Evapotranspiration," Earth Interact., vol. 15, no. 1, pp. 1-14, Jan. 2011. https://doi.org/10.1175/2010EI363.1

[71] A. F. Carril et al., "Performance of a multi-RCM ensemble for South Eastern South America," Clim. Dyn., vol. 39, no. 12, pp. 2747-2768, Dec. 2012. https://doi.org/10.1007/s00382-012-1573-z

[72] M. Llopart; R. P. da Rocha; M. Reboita; S. Cuadra, "Sensitivity of simulated South America climate to the land surface schemes in RegCM4," Clim. Dyn., vol. 49, no. 11-12, pp. 3975-3987, Feb. 2017. https://doi.org/10.1007/s00382-017-3557-5 